\title{
SilverStar-Förderpreis
}

\section{Graswurzelprojekte für Diabetespatienten erhalten 25000 Euro}

\section{Anlässlich der 6. Herbsttagung der Deutschen Diabetes Gesellschaft wur- de nun zum zweiten Mal der SilverStar Förderpreis verliehen. Drei Projekte wurden ausgezeichnet.}

Der von Berlin-Chemie gestiftete Preis wird jährlich vergeben. Ziel ist es, Initiativen zu fördern, die Patienten mit Diabetes im Alltag unterstützen. Beworben haben sich über fünfzig Projektgruppen. Die Förderanträge wurden von einer Jury bewertet, der unter anderem der Diabetologe Prof. Rüdiger Petzoldt und der Geriater Prof. Cornel Sieber angehörten.

Der erste Preis (12500 Euro) ging an das Projekt "Gemeindeschwester Muschenheim". Die Bürger von Muschenheim im Städtchen Lich haben einen Verein gegründet, der über Mitgliedsbeiträge zwei Gemeindeschwestern finanziert. Sie kümmern sich in Kooperation mit den jeweiligen Ärzten zu festgelegten Sprechzeiten um die Betreuung älterer Menschen und speziell um Diabetes- patienten. Zu den Aufgaben der Gemeindeschwestern, die selbst Teil der Dorfgemeinschaft sind, gehören Therapiekontrollen und Blutzuckermessung, aber auch Schulungen, persönliche Zuwendung und das Lösen von Alltagsproblemen.

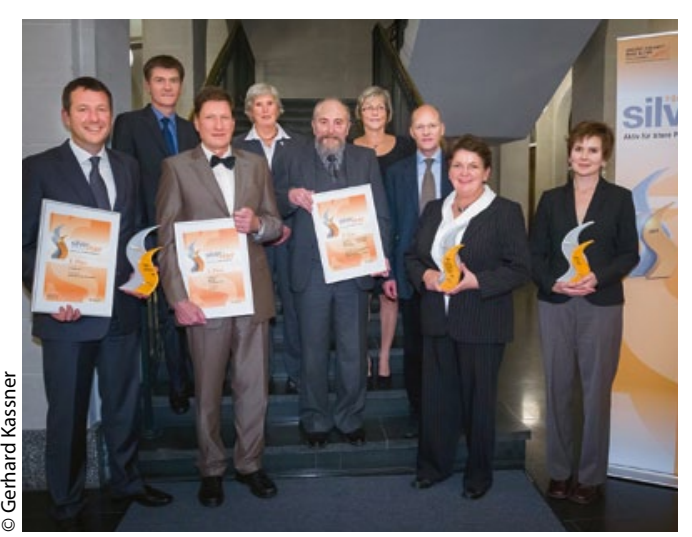

Die SilverStar Preisträger 2012 (von links nach rechts): Dr. Detlef Kuhn, Torsten Flöttmann, Dr. Michael Herr, Dr. Ortrud Hamann, Peter Grau, Hilde Herr, Prof. Cornel Sieber, Irene Feucht, Marie-Louise Thiel.
Der zweite Preis (7500 Euro) ging an das Projekt „Pro Edith“ am Marienkrankenhaus in Schwerte. Dort wurde ein fachabteilungsübergreifendes Diabetesteam gebildet. Es begleitet Patienten mit der Nebendiagnose Diabetes während des stationären Aufenthalts und übernimmt koordinierende Funktionen beim Übergang in die ambulante Betreuung.

Ebenfalls mit einem zweiten Preis (5000 Euro) ausgezeichnet wurde eine Initiative des Agaplesion Bethesda Krankenhauses Stuttgart. In dieser Klinik erhalten Diabetiker noch während des stationären Aufenthalts eine Essensunterlage, auf der der richtige Umgang mit der Insulintherapie grafisch und in Worten dargestellt ist. Diese Unterlage können die Patienten mit nach Hause nehmen und haben die wichtigsten Informationen so immer zur Hand.

(gvg) ID

|| www.silverstar-preis.de

\section{Herzinsuffizienz}

\section{Fast jeder Zweite ist ein Kandidat für Frequenzreduktion}

Für nahezu jeden zweiten Patient mit Herzinsuffizienz wäre der $\mathrm{I}_{\mathrm{f}}$-Kanalblocker Ivabradin eine Therapieoption, so das Ergebnis einer großen Screeningstudie.

Die Herzinsuffizienztherapie hat sich in den letzten Jahren verbessert; ACE-Hemmer, Betablocker und Aldosteron-Antagonisten lassen die Patienten länger leben. Doch wenn die Blutdrucksenkung ausgeschöpft ist, müssen andere Wirkprinzipien greifen. Die Herzfrequenz zu reduzieren, ist ein Ansatzpunkt. Sie hat sich als unabhängiger prognostischer Faktor herausgestellt: „Fünf Schläge pro Minute mehr bedeuten ein 16\% höheres kardiovaskuläres Risiko für Hospitalisierung und Tod", verdeutlichte Prof. Michael Böhm, Universität Homburg/Saar. Der If-Kanal-Blocker Ivabradin (Procoralan ${ }^{\circledR}$ ) re- duziert bei Patienten im Sinusrhythmus selektiv die Herzfrequenz, ohne wie Betablocker die Hämodynamik zu beeinflussen. Dieses Therapieprinzip ist bei KHK v.a. symptomatisch und bei Herzinsuffizienz prognostisch wirksam. Letzteres hat die SHIFTStudie ergeben: Die herzinsuffizienzbedingte Mortalität und Hospitalisierungsrate von 6558 Patienten wurde jeweils um 25\% signifikant reduziert. Je stärker die Ruhefrequenz gesenkt wurde, desto deutlicher verbesserte sich auch die Lebensqualität.

Die Ergebnisse haben sich auch auf die ESC-Leitlinien ausgewirkt: Diese empfehlen den Einsatz von Ivabradin bei chronischer systolischer Herzinsuffizienz mit eingeschränkter Pumpfunktion und Sinusrhythmus dann, wenn trotz Betablocker die Herzfrequenz über 75/min liegt (Ila-Empfehlung) oder eine Kontraindikation bzw. eine Intole- ranz gegen Betablocker vorliegt (Ilb-Empfehlung).

Erste Ergebnisse einer groß angelegten Screeningstudie (INDICATE) quantifizieren, für wie viele Herzinsuffiziente Ivabradin eine Therapieoption wäre. Die Mehrheit der 15148 Patienten befand sich in den NHYAKlassen II und III und war im Sinusrhythmus, jeder vierte wies eine eingeschränkte Pumpfunktion unter $35 \%$ auf, 49,5\% nahmen 50 $100 \%$ der von Leitlinien empfohlenen Betablocker-Dosis ein, und $53 \%$ hatten eine Frequenz von über 75 Schlägen pro Minute. Damit ist etwa jeder zweite Patient ein Therapiekandidat, erklärte Prof. Christian Zugck, Heidelberg.

(vsc) II

II Symposium, DGK-Herbsttagung, Hamburg, 12. Oktober 2012 (Veranstalter: Servier) 